\title{
Desarrollo de comunidades rurales y degradación de recursos forestales en la región Occidente de México
}

\author{
Development of rural communities and forest degradation in Western Mexico
}

\section{Resumen}

Entre las medidas más aceptadas para disminuir las concentraciones de gases efecto invernadero (y que contribuyen al cambio climático) está la extensión de recursos forestales y el aumento en el volumen en pie de los bosques. Estas medidas a menudo imponen restricciones sobre el uso de los recursos naturales de la población rural que depende directamente de los recursos forestales al ser su medio de subsistencia. Para evitar una disminución del bienestar de la población local producida por las limitaciones tras implementar un programa de conservación es importante conocer las condiciones sociales y económicas de estas comunidades y también de los factores que pueden estar influyendo en la rentabilidad de actividades económicas actuales que producen degradación forestal. Este estudio calcula los beneficios netos de la práctica de estas actividades en la región occidente de México y estima el valor de sus componentes mediante una regresión hedónica. El análisis indica que los factores que influyen en la rentabilidad de estas actividades que degradan los recursos forestales están relacionadas con prácticas de agricultura rudimentaria, realizada mayormente por la población más pobre y que carece de técnicas modernas de cultivo. Además, se propone que el financiamiento de organismos internacionales que incentivan el aumento de las masas forestales y son una fuente de desarrollo económico deberían identificarse como un complemento a las iniciativas de gobiernos locales y no como la fuente responsable de promover el desarrollo de las comunidades rurales.

Palabras clave:

- Degradación Forestal

- Agricultura migratoria

- Recursos forestales

- Beneficios netos

\section{Abstract}

Between the measures most accepted to diminish the gas concentrations greenhouse effect (and that contribute to the climate change) there is the extension of forest resources and the increase in the volume in foot of the forests. These measures often impose restrictions on the use of the natural resources of the rural population who depends directly on the forest resources to the being his way of subsistence. To avoid a decrease of the well-being of the local population produced by the limitations after implementing a program of conservation is important to know the social and economic conditions of these communities and also of the factors that can be influencing the profitability of economic current activities that produce forest degradation. This study calculates the net profits of the practice of these activities in the region west of Mexico and estimates the value of his components by means of a regression hedónica. The analysis indicates that the factors that influence the profitability of these activities that degrade the forest resources are related to practices of rudimentary agriculture, realized mainly by the poorest population and that lacks modern technologies of culture. In addition, he proposes that the international organizations' financing that they stimulate the increase of the woodlands and they are a source of economic development they should be identified as a complement to the initiatives of local governments and not as the responsible source of promoting the development of the rural communities.

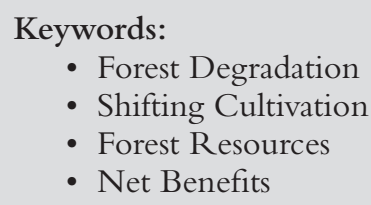

JEL: O13, O18, Q12, R11

\footnotetext{
* Doctora en Economía Aplicada por la Univesidad Autónoma de Barcelona. Actualmente es profesora en la Facultad de Economía de la UNAM, donde actualmente hace una estancia posdoctoral.armoniab@gmail.com. ** Ing. en Recursos Naturales y Agropecuarios por la Universidad de Guadalajara. Actualmente es investigadora en la Secretaría de Medio Ambiente y Desarrollo Territorial.
} 


\section{Introducción}

Los cambios en las temperaturas, la pérdida de biodiversidad, el aumento en la intensidad y frecuencia de desastres naturales, la erosión del suelo, la degradación de los recursos forestales, entre otros, son ejemplos de algunas preocupaciones ambientales que han surgido en los últimos años en diferentes países. A menudo la presencia de estos fenómenos se relacionan directamente con el aumento de emisiones de los gases efecto invernadero producidas por las actividades humanas. Para contrarrestar estos impactos negativos surgen iniciativas nacionales e internacionales con el objetivo de reducir las emisiones en la atmosfera (principalmente dióxido de carbono - $\mathrm{CO} 2-$ ) y se proponen medidas para mejorar la cantidad y calidad de recursos naturales de los países como una forma de mitigar los efectos negativos de los cambios en las temperaturas (Protocolo de Kyoto, REDD+, CDM, PES, VCM). Entre las medidas que más se promueven para ayudar a detener el efecto calentamiento esta la extensión de los recursos forestales (o disminución en las tasas de deforestación) y el aumento en el volumen en pie de los bosques (o disminución de degradación) porque se asume que la disminución de la superficie forestal podría resultar en un aumento del nivel de carbono emitido a la atmosfera, propiciando el efecto calentamiento.

Paradójicamente la evidencia empírica parece mostrar que este creciente interés internacional por disminuir la degradación ambiental viene acompañado por un aumento en la intensidad y el uso de los recursos naturales, principalmente de un sector de la población de países en desarrollo. Citando una de las estadísticas mas referidas en las evaluaciones de los recursos forestales es la pérdida neta de área de bosque, se tiene que entre los años 1990 y 2005 la perdida de área forestal en el mundo fue de $3.2 \%$, un área equivalente a $\mathrm{Pa}-$ namá, Dinamarca y Estonia (FAO, 2010). No es coincidencia que América del Sur y África hayan registrado las mayores pérdidas netas en aéreas de bosque, porque en esas regiones millones de personas que tienen acceso a recursos forestales y dependen directamente de ellos como medio de subsistencia. Con frecuencia la urgencia por cubrir necesidades básicas como la alimentación y vivienda son prioridades para la población y los gobiernos de estos países en comparación a otros intereses como la protección del medio ambiente. Las estadísticas ponen de relieve el hecho de que el entorno social y cultural de las comunidades locales de algunos países en desarrollo está estrechamente vinculado a actividades de consumo directo de los recursos naturales. 
Para alcanzar su objetivo, los programas de conservación internacionales suelen limitar el patrón habitual de consumo de recursos naturales que podrían dar lugar a una disminución del bienestar de la población local, especialmente si la rentabilidad obtenida por realizar actividades de conservación no se corresponden con la rentabilidad esperada antes de implementar el programa de conservación propuesto. Pese a estas circunstancias, las iniciativas internacionales continúan financiando programas de conservación que puedan mejorar el bienestar de poblaciones locales, intentando crear vínculos entre el desarrollo económico de las comunidades locales y una actitud de cuidado ambiental.

México no es una excepción en la imagen que predomina en los países en desarrollo. En México una tercera parte de la superficie está cubierta por bosques (64 802000 hectáreas) y 70\% son propiedad de comunidades locales (ejidos). Entre 1990 y 2000 México registró una disminución de superficie forestal anual de $0.52 \%$ y entre el 2000 y 2005 una disminución anual de $0.40 \%$ (FAO, 2010). Ante la divergencia entre las iniciativas internacionales y las decisiones o acciones de las comunidades locales es posible que las nociones de la degradación de bosques de las comunidades locales y de los organismos globales puedan diferir. De esta manera, las comunidades que dependen directamente de los bosques y selvas pueden percibir un bosque en estado degradado como aquel que les proporciona beneficios directos y prefieran mantenerlo en esas condiciones. Así, el consumo de leña o de materiales para construcción como los postes podría estar relacionado con la degradación del bosque porque su extracción disminuye la biomasa y la cobertura de masa forestal. Es conocido que la práctica de agricultura permanente contribuye a la deforestación de bosques porque implica la tala de áreas forestadas para cambiar el uso del suelo. Sin embargo, la práctica de agricultura itinerante o migratoria, que se explicara en detalle en la sección de contexto de caso de estudio, puede estar asociada a la degradación de selvas y bosques y ha sido menos estudiada en el área de investigación científica (en este estudio los términos agricultura itinerante y migratoria se usaran indistintamente). Estas decisiones que implican un cambio en el uso del suelo, y que pueden producir degradación del medio ambiente, pueden estar afectadas por factores sociales, económicos y ambientales; por tanto, un conocimiento sobre las condiciones locales pueden ayudar a entender por qué las comunidades practican algunas actividades que contribuyen a la degradación de los recursos naturales y si existen soluciones potenciales para mitigar la degradación del medio ambiente. A la vez, la población con abundantes recursos forestales suelen estar relacionados a condiciones de alta marginalidad 
y pobreza pese a estar rodeados de un entorno rico en biodiversidad y recursos naturales (Wunder, S., 2001). Cabe preguntarse cuáles son los factores que influyen en las decisiones de los campesinos al practicar actividades que aumentan el deterioro de los recursos forestales y si potencialmente existe algún mecanismo (incentivos) que podría modificar su comportamiento.

En este estudio se identifican las variables que influyen en la rentabilidad de la agricultura itinerante y permanente y se comparan con los beneficios ambientales propuestos por el programa de pagos por servicios ambientales (PSA). Tras esta introducción, se presenta la metodología usada para los cálculos de los beneficios netos. En lo que sigue, se describe la organización de los campesinos de la Cuenca del Río Ayuquila, Jalisco y también presenta el contexto del caso de estudio. Tras ello, se presentan los resultados de la aplicación del método y la última sección presenta la discusión y algunas observaciones finales.

\section{Metodología}

La teoría económica proporciona el fundamento para la maximización del bienestar de los individuos partiendo del supuesto de que los individuos se comportan racionalmente y que intentan maximizar su utilidad (Miller, 2006) y este es el fundamento usado para el análisis de este estudio. Si los individuos maximizan su utilidad, pueden elegir el nivel de consumo de los recursos naturales en su entorno, es decir, aquel consumo que maximiza su utilidad, sujeto a un presupuesto limitado (ingresos, tiempo). En este caso, existe una variedad de recursos naturales que podemos denotar como $n$, asumiendo un numero finito de recursos naturales o bienes. Los recursos naturales se denotan como $l$ donde $y_{l}$ será el tiempo.

La decisión de extraer una variedad de recursos naturales puede representarse con un vector de $n$ componentes. Si un consumidor elige una combinación especifica de consumo $y=\left[y_{1}, y_{2}, \ldots y_{l}, \ldots y_{n}\right]$ indica que el individuo obtiene $y_{1}$ beneficios al extraer una cantidad de recursos, por ejemplo, la cantidad de leña, $y_{2}$ le proporcionara beneficios al extraer postes para cercar su terreno, etc. Para efectos de este estudio se elimina la posibilidad de elecciones de consumo que arrojen componentes negativos, así las alternativas disponibles de los individuos se compone de vectores de $n$ recursos naturales donde cada componente es positivo y lo podemos denotar como $R_{+}^{n}$. Una vez presentadas las posibles combinaciones de consumo disponibles para el individuo se define el punto optimo que cada individuo elegirá entre todas las 
opciones. Las preferencias de los individuos se pueden representar como una función de utilidad $U(y, w)$ que es creciente en $y$ y decreciente en $w$, atribuido al efecto cansancio de consumir una determinada especie de un recurso natural y siendo cuasi-cóncavas en estas variables; así, y dependerá de las características físicas del recursos natural. Si los beneficios son crecientes y cóncavos en $q$ cuando los atributos asociados a $n$ recursos naturales son elegidos de acuerdo a las preferencias del individuo $y=f(q)$, decrecientes y convexos en $q$ para aquellos recursos naturales asociados $n$ que son menos preferidos de acuerdo a las preferencias $w(q)$.

Los individuos elegirán de acuerdo a $\max _{y} U(y, w(y))$ sujetos a $y=f(q)$ donde $q=\left(q_{1}, q_{2}, \ldots q_{l}, \ldots q_{n}\right)$ es el vector de características de cada recurso natural. La decisión optima de consumo depende de las características de los $n$ recursos naturales, La función que mide la proporción de beneficios obtenidos de acuerdo a cada recurso natural consumido vendrá dada por $y^{*}(q)$. $y^{*}(q)=\left[y_{1}(q), y_{2}(q), \ldots y_{l}(q), \ldots y_{n}(q)\right]$.

Para evaluar las características de la tierra que influyen en la obtención de beneficios por la práctica de agricultura itinerante se ha aplicado una ecuación hedónica, o por componentes. Los modelos hedónicos se han usado con frecuencia para determinar los precios implícitos (o de los componentes) y su impacto en la productividad de la agricultura (Brown and Barrows, 1985; Ervin and Mill, 1985; Mendelsohn et al., 1994; Dinar et al., 1998; Evenson and Alves, 1998). Para calcular los beneficios netos de acuerdo a la práctica de agricultura se diseñó un cuestionario para investigar el consumo de los recursos naturales, su frecuencia de uso, temporada y precios de mercado de cada bien o servicio, tamaño de parcelas, períodos de descanso de la tierra, producción obtenida, etc. De esta manera e elaboró un inventario detallado con el registro de las decisiones reales (preferencias) de los campesinos según sus prácticas de agricultura. En la literatura este método basado en la observación de las preferencias reales se conoce como Técnica de Comportamiento Observado (Adamowicz, et al., 1997) o método a Precios de Mercado (IIED, 2003).

\section{Contexto del caso de estudio}

En México casi una tercera parte de los bosques y selvas son propiedad de comunidades o ejidos. La dotación de tierras se otorgó por resolución presidencial a núcleos de población desde la segunda mitad de la década de 1930 y posteriormente se otorgaron ampliaciones de terreno. La idea de la repartición 
era la formación de centros agrícolas competitivos con capacidad de autosuficiencia alimentaria . Las comunidades tendrían la máxima autoridad sobre las tierras que les fueron otorgadas y podrían usufructuarlas de acuerdo a lo que se estableciera en una Asamblea conformada por la mayoría de los ejidatarios o comuneros (como se le conoce a cada miembro del grupo con derecho a voz y voto). Una de las características más relevantes de los ejidos era que ninguno podía vender su posesión sino solo podía ser transferido a un familiar directo como sucesor con aprobación ante la Asamblea. Estas políticas sociales buscaban el desarrollo de las comunidades rurales otorgando poder de pleno uso de los recursos naturales, a su vez, favoreciendo el deterioro de los recursos forestales al ser los bosques y selvas la principal fuente de ingresos para los campesinos.

Tras la Reforma al Artículo 27 Constitucional en 1992 el Gobierno Federal implementó el Programa de Certificación de Derechos (PROCEDE), con lo que ejidatarios y comuneros pudieron delimitar legalmente las parcelas y obtener un Certificado de Derechos Agrarios, dando posibilidad de venta de tierras ejidales o comunales. Así, la reforma convierte las tierras de propiedad social en propiedad privada individual y acredita a ejidatarios como poseedores de su parcela, por ende, les permite transferir, ceder o vender tales derechos a terceros.

Desde su origen, las tierras ejidales generalmente se dividen en áreas cerriles (para uso común de todo el grupo) y en áreas planas divididas en parcelas (para cada ejidatario); en algunos ejidos también se destinaron áreas para asentamientos humanos. El área cerril se suele dejar en propiedad común con la finalidad de que todos los ejidatarios y sus familias puedan obtener beneficios como la extracción de frutos, leña, postes para cercado o construcciones, aunque a veces se requiere permiso escrito por parte del comisariado ejidal (Presidente, Secretario y Tesorero electos en Asamblea). En la época de lluvia el área de uso común también es usado como agostadero, donde el ganado se apacentará mientras termina el ciclo de los cultivos agrícolas. Aunque el reparto individual de las áreas de uso común no puede autorizarse legalmente, algunos ejidos decidieron hacerlo así, en acuerdo interno y porque así convenía a sus intereses.

En la zona de estudio se sigue este orden y la actividad agrícola se practica principalmente bajo dos sistemas de cultivo: la más habitual se hace en parcelas de áreas planas que pueden depender del temporal de lluvia o tener sistema de riego. En el último caso, se puede disponer de agua durante todo el año y obtener dos ciclos de producción anual. En la forma de agricultura permanente por lo general se cultiva maíz, aunque en los últimos años la 
producción agrícola se ha diversificado. La mayor parte es sembrada por los propietarios de las parcelas pero existen casos en que el poseedor opta por rentar la tierra y es el arrendador quien la trabaja. El otro sistema de cultivo es la agricultura migratoria o itinerante, una práctica rudimentaria que tiene un ciclo al año y se hace bajo sistemas poco tecnificados porque las superficies de siembra se ubican generalmente en áreas de uso común, donde predominan laderas o pendientes moderadas y son conocidas localmente como coamiles en los que se produce principalmente maíz y a veces calabaza y frijol simultáneamente. La apertura de un coamil muchas veces implica el desmonte de superficie arbolada. Esta práctica la desarrollan principalmente los campesinos que no tienen acceso a sistemas de riego y la siembra depende de la lluvia, algunos son poseedores de la tierra pero otros siembran en superficies prestadas con la condicionante de dejar al poseedor la pastura restante después de la cosecha. Los coamiles son cultivados por dos o tres años consecutivos y después son abandonados para dejar que se regenere y crezca la vegetación de manera natural por unos cinco años. Esta práctica se hace con la finalidad de que la tierra descanse y recupere sus nutrientes, cuidando que no se llegue a cubrir de árboles totalmente porque en ese caso ya no se les permitirá cortarlos e incurrirían en delito.

Entre tanto que un coamil se deja en descanso, los campesinos estarán cultivando otra superficie descansada, eliminando la vegetación que haya crecido allí (a este proceso le llaman barbecho). El ciclo de cultivo en coamil inicia en los meses de estiaje entre abril y mayo. Los restos del ciclo anterior o el material vegetal desmontado según sea el caso, se amontonan y se queman para que la ceniza se reincorpore al suelo y facilite la siembra que será al iniciar los primeros dos meses de lluvia (junio-julio). En el mes de Agosto los campesinos continúan con la remoción de maleza y fertilización, para lo cual generalmente se utilizan insumos agroquímicos en algunos casos y productos orgánicos o tradicionales (en los últimos años se está promoviendo por parte de universidades y dependencias de gobierno la incorporación de la materia orgánica al suelo para evitar la quema y prevenir incendios forestales). Si el temporal de lluvias es suficiente, la cosecha tendrá lugar en Noviembre o Diciembre. Ya terminada la cosecha los coamiles serán ocupados por el ganado que comerá los restos del cultivo, hasta iniciar el nuevo ciclo de siembra. La cosecha de un coamil con frecuencia es menor a la cosecha que se obtiene de las parcelas de agricultura convencional. 
La agricultura itinerante o migratoria provee sustento para muchos campesinos en la zona de estudio y su práctica también es frecuente en zonas rurales de otros países en desarrollo. A menudo es un sistema que está relacionado con la degradación ambiental, la emisión de gases efecto invernadero, perdida de biodiversidad y erosión del suelo. En el área de estudio un poco más de la mitad de los campesinos entrevistados tenia o había cultivado un coamil (o agricultura itinerante).

\section{Muestra}

Se entrevistó a una muestra aleatoria de campesinos mayores de edad en diez municipios situados en la Cuenca de Ayuquila, Jalisco en el verano de 2012. En total se realizaron 330 entrevistas que fueron representativas en términos del tamaño del ejido de residencia y tipo de propiedad (propietarios, no propietarios, ejidatarios y comuneros). El porcentaje de respuestas al cuestionario fue de $95 \%$ y la modalidad de la entrevista fue personal, realizadas en los domicilios de los campesinos o en sus tierras de trabajo. El cuadro 1 muestra las características socio-demográficas de los entrevistados.

De acuerdo a las estadísticas de INEGI (2010) el tamaño de hogar de la muestra entrevistada se corresponde con la de registrada para la población total. Otras variables como educación y genero no son representativas en relación a la población total. La muestra entrevistada ha recibido menor educación formal en comparación al promedio de la población total y la representación de hombres entre los entrevistados deja ver que las decisiones en el campo recaen mayormente en los hombres, que son titulares legales de la tierra. No ha sido frecuente encontrar mujeres de mediana edad entre los titulares de la tierra, en general, el fallecimiento del cónyuge significa el paso de la propiedad a la esposa.

Una comparación entre las características de campesinos que usan sistema de agricultura migratoria y permanente muestra que los primeros dependen más de combustibles como la leña para cocinar respecto a los practican agricultura permanente. También se distingue la variable de superficie sembrada, en la que el numero de hectáreas de agricultura permanente casi dobla la cantidad promedio de hectáreas sembradas en sistema de agricultura migratoria. 


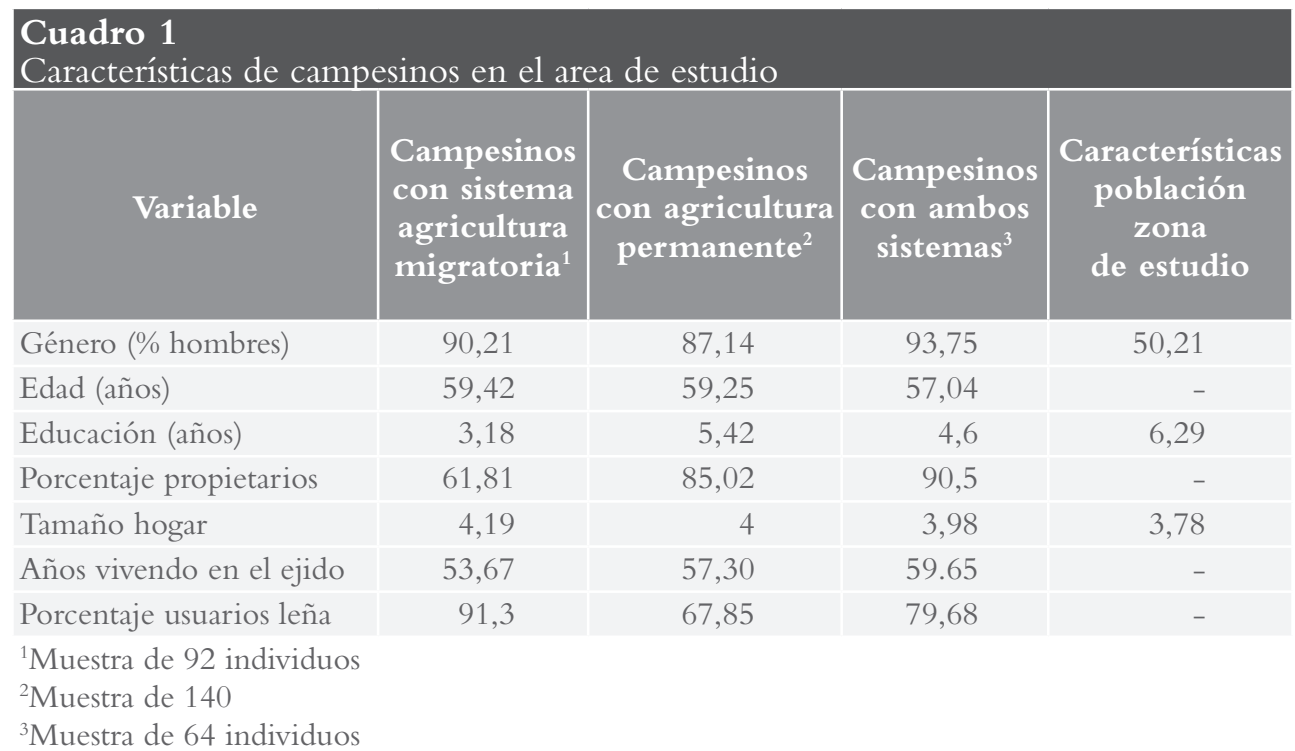

\section{Resultados}

Casi un tercio de los entrevistados correspondieron a la práctica de agricultura itinerante (31\%), aproximadamente la mitad de la muestra se dedicaba únicamente a agricultura permanente $(47 \%)$ y el resto sembraba haciendo una combinación de los dos sistemas de agricultura (22\%). En el cálculo de beneficios netos se excluyeron de la muestra aquellos individuos que practicaban otro tipo de cultivo distinto al maíz (agave, aguacate, caña) porque los ciclos de cultivo, sus costes y el patrón de consumo de recursos naturales difieren en comparación a los registrados por los grupos de campesinos que siembran de maíz. Siguiendo el marco teórico presentado en la sección de metodología, el cálculo de beneficios netos incluyen las siguientes variables: beneficios obtenidos por agricultura (según cada sistema), extracción de leña, extracción de postes para construcción, cantidad de ganado y extracción de frutos y plantas medicinales.

De acuerdo a los estadísticos descriptivos del cuadro 2 los beneficios netos de los individuos que practican agricultura itinerante son los más bajos de las tres categorías. Según los datos, un campesino bajo sistema de agricultura migratoria obtiene un beneficio neto de 20150 pesos anuales (incluyendo el consumo de otros recursos naturales) con una desviación estándar de 14600 pesos. En comparación, las decisiones y prácticas de consumo de los campesinos que siembran bajo un sistema de agricultura permanente doblan los 
beneficios netos medios obtenidos por grupo que siembra en un sistema de agricultura migratoria (incluyendo el consumo de recursos naturales). También se observa una diferencia en la superficie media anual sembrada para cada grupo: la media sembrada en agricultura migratoria es de 3 hectáreas mientras que para agricultura permanente la media es de casi 6.5 hectáreas por individuo y en promedio, los campesinos de agricultura itinerante reciben menos subsidios que los de agricultura permanente. En parte, esta diferencia en las subvenciones recibidas se debe a que el subsidio está condicionado que la tierra registrada sea cultivada cada año, y como se ha explicado, el sistema de agricultura itinerante no sigue este patrón sino que se dejan en descanso durante algún período que puede ser variable. Otra característica relevante es el uso de caballo o mula para la siembra; los campesinos con agricultura itinerante usan con mayor frecuencia algún animal en sus prácticas de agricultura, mientras que el sistema de agricultura permanente es mas tecnificado mediante el uso de tractores. Esta característica está directamente relacionada con la inclinación de la tierra; el primer grupo suele sembrar en áreas más inclinadas o con pendientes, donde difícilmente podrían hacer uso de tractor y los segundos siembran en tierras llanas, superficies donde pueden hacer uso de tractor.

\begin{tabular}{|c|c|c|c|c|c|c|}
\hline $\begin{array}{l}\text { Cuadro } 2 \\
\text { Definición }\end{array}$ & variables & stadísticos & escriptivo & & & \\
\hline Variable & Agricultu & Itinerante & $\begin{array}{l}\text { Agri } \\
\text { Perm }\end{array}$ & $\begin{array}{l}\text { Itura } \\
\text { nente }\end{array}$ & $\begin{array}{r}\text { Dos si } \\
\text { agri }\end{array}$ & $\begin{array}{l}\text { mas de } \\
\text { ltura }\end{array}$ \\
\hline & Media & $\mathrm{SD}$ & Media & SD & Media & SD \\
\hline Ben_Net ${ }^{1}$ & 20143.2 & 14586.9 & 81735.5 & 72500.7 & 63566.1 & 42362.1 \\
\hline Postes $^{2}$ & 41.43 & 76.95 & 69.26 & 132.12 & 74.69 & 89.23 \\
\hline Ganado $^{3}$ & 3.25 & 6.63 & 6.50 & 13.37 & 16.55 & 16.92 \\
\hline Distancia $^{4}$ & 7.47 & 8.43 & 3.67 & 2.93 & 6.67 & 5.22 \\
\hline Bestia $^{5}$ & 0.58 & 0.40 & 0.36 & 0.87 & 1.06 & 1.31 \\
\hline Hectáreas $^{6}$ & 3.19 & 3.14 & 6.37 & 4.73 & 13.20 & 10.39 \\
\hline Expe $^{7}$ & 38.64 & 16.84 & 39.34 & 17.03 & 37.36 & 16.23 \\
\hline Genero $^{8}$ & 0.90 & 0.30 & 0.87 & 0.34 & 0.94 & 0.24 \\
\hline Subvención ${ }^{9}$ & 0.37 & 0.48 & 0.69 & 0.46 & 0.71 & 0.46 \\
\hline Coste $^{10}$ & 14687.10 & 20930.70 & 54024.40 & 50617.20 & 48184.50 & 30987.00 \\
\hline Pendiente ${ }^{11}$ & 1.92 & 0.27 & 1.26 & 0.44 & 1.84 & 0.37 \\
\hline $\begin{array}{l}{ }^{1} \text { Beneficios } n \\
{ }^{3} \text { Cabezas de } \\
{ }^{6} \text { Promedio he } \\
\text { bre, } 0 \text { en caso } \\
{ }^{10} \text { Coste por }\end{array}$ & $\begin{array}{l}\text { os en pesos } \\
\text { ado; }{ }^{4} \mathrm{Kilóm} \\
\text { áreas sembra } \\
\text { ntrario; }{ }^{9} \mathrm{Va}\end{array}$ & $\begin{array}{l}2012 ;{ }^{2} \text { Núme } \\
\text { os; }{ }^{5} \text { Número } \\
;{ }^{7} \text { Años de es } \\
\text { le dicotómi }\end{array}$ & $\begin{array}{l}\text { o de postes } \\
\text { de caballos } \\
\text { periencia; }{ }^{8} \\
\text { indicando }\end{array}$ & $\begin{array}{l}\text { traídos en } \\
\text { dos para se } \\
\text { iable dicot } \\
\text { si recibe sul } \\
{ }^{11} \text { Pendient }\end{array}$ & $\begin{array}{l}\text { año; } \\
\text { orar; } \\
\text { ica indican } \\
\text { nción, } 0 \text { en }\end{array}$ & $\begin{array}{l}1 \text { si es hon } \\
\text { so contrari } \\
\text { lel mar. }\end{array}$ \\
\hline
\end{tabular}


Para la evaluación de los factores que influyen en la rentabilidad de sistemas de agricultura que contribuyen en la degradación de bosques o selvas se incluyeron todas aquellas variables que podrían afectar a los beneficios netos (variable dependiente) del sistema de agricultura migratoria, por ejemplo: la extracción de postes que se usan para delimitar las tierras o parcelas, el uso de caballo o tractor, la cantidad de ganado, la experiencia del campesino en el sistema de agricultura, la obtención de subvención para sembrar, la cantidad de hectáreas sembradas y el sexo de la persona. Otras variables físicas como la distancia del hogar a la tierra de trabajo y la elevación de la tierra también fueron incluidas en las ecuaciones hedónicas para cada sistema de agricultura.

El cuadro 3 recoge los resultados de la estimación de los tres modelos, donde todas las variables son significativas al menos en un nivel de 5\%. Para los tres tipos de productores un aumento de una unidad en la cantidad de ganado genera, manteniendo todo lo demás constante, un aumento en el beneficio neto entre 1050 y 1600 pesos. Como se esperaba, el estimador de la variable Hectáreas muestra que la superficie sembrada es un factor directo que influye en los beneficios netos de la agricultura itinerante y también lo es la experiencia que el campesino tenga en este sistema de agricultura. Se probaron otras variables en el modelo pero ninguna resultó significativa, sugiriendo que este tipo de agricultura sigue una práctica rudimentaria con poca o ninguna tecnología. No influye la distancia entre el hogar de los campesinos y las tierras, que en promedio se sitúan a 7.5 kilómetros, tampoco influye la extracción de postes posiblemente porque el uso de postes no es para uso personal sino para venta. Aun cuando el uso de caballos era frecuente entre este grupo, la variable no resultó significativa. El segundo modelo corresponde a los factores que influyen en los beneficios netos de la agricultura permanente. En las entrevistas estos productores enfatizaban la importancia de usar postes para construir vallas para delimitar sus terrenos e impedir el paso del ganado que podría afectar la siembra, variable que ha resultado significativa a 5\%, indicando que por cada poste usado tiene un efecto positivo en el beneficio neto. Como se esperaba, una mayor distancia entre el hogar del campesino y la tierra de cultivo influye negativamente en el beneficio neto de los productores y también el uso de un caballo o mula, por ese motivo la mayoría hacía uso de tractor. Finalmente, cada unidad monetaria invertida en la producción en promedio genera beneficios netos de 0.84 pesos, manteniendo todo lo demás constante. Los beneficios netos de los agricultores que combinan los dos sistemas en distintas superficies aumentan cuando se hace uso de alguna bestia para sembrar y cuando la experiencia de sembrar es mayor. Para este grupo 
el recibir una subvención aumenta sus rendimientos netos y también el factor genero (ser hombre). Como es esperado, cuando la siembra se hace principalmente en laderas o pendientes muy pronunciadas disminuye los beneficios.

\begin{tabular}{|c|c|c|c|}
\hline \multicolumn{4}{|c|}{$\begin{array}{l}\text { Cuadro } 3 \\
\text { Resultados de la regresión Mínimos Cuadrados Ordinarios }\end{array}$} \\
\hline Variable & $\begin{array}{l}\text { Agricultura } \\
\text { Itinerante }\end{array}$ & $\begin{array}{l}\text { Agricultura } \\
\text { Permanente }\end{array}$ & $\begin{array}{l}\text { Dos sistemas de } \\
\text { agricultura }\end{array}$ \\
\hline Constante & $\begin{array}{l}9826.77^{* * *} \\
(.0035)\end{array}$ & $\begin{array}{l}2952.43 \\
(.8007)\end{array}$ & $\begin{array}{c}1310.10^{* *} \\
(.0316)\end{array}$ \\
\hline Postes & . & $\begin{array}{c}50.0491^{* *} \\
(.0312)\end{array}$ & - \\
\hline Ganado & $\begin{array}{l}1555.57^{* * *} \\
(.0001)\end{array}$ & $\begin{array}{c}1663.00^{* * *} \\
(.0014)\end{array}$ & $\begin{array}{c}1055.65^{* *} \\
(.0433)\end{array}$ \\
\hline Distancia & 1.0001 & $\begin{array}{l}-47.9836^{* * *} \\
(-.0001)\end{array}$ & 1.07007 \\
\hline Bestia & - & $\begin{array}{c}-13733.9^{*} \\
(-.0503)\end{array}$ & $\begin{array}{l}17480.1^{* *} \\
(.0126)\end{array}$ \\
\hline Hectáreas & $\begin{array}{c}1505.57^{* *} \\
(.0487)\end{array}$ & $\begin{array}{c}3126.03^{* * *} \\
(.0418)\end{array}$ & - \\
\hline Expe & $\begin{array}{c}28.4525^{*} \\
(.0697)\end{array}$ & - & $\begin{array}{c}65.5855^{*} \\
(.0720)\end{array}$ \\
\hline Genero & - & - & $\begin{array}{c}73364.8^{* *} \\
(.0372)\end{array}$ \\
\hline Subvención & - & - & $\begin{array}{c}143.556^{* * *} \\
(.0047)\end{array}$ \\
\hline Coste & - & $\begin{array}{c}0.8419^{* * * *} \\
(.0000)\end{array}$ & $\begin{array}{c}.32375^{* * *} \\
(.0000)\end{array}$ \\
\hline Pendiente & - & ! & $\begin{array}{c}-3905.26^{*} \\
(-.0859)\end{array}$ \\
\hline $\mathrm{R}^{2}$ & 0.25 & 0.48 & 0.49 \\
\hline $\mathrm{n}$ & 92 & 140 & 64 \\
\hline
\end{tabular}

Valores del estadístico t en paréntesis

* Significativo para un nivel de error máximo del 10\%

** Significativo para un nivel de error máximo del 5\%

*** Significativo para un nivel de error máximo del 5\%

De esta forma, cada grupo se ve afectado por variables específicas si embargo, el grupo que practica el sistema de agricultura frecuentemente relacionado con la degradación de recursos forestales (i.e. agricultura itinerante) es el sistema más rudimentario y menos afectado por variables físicas. Por ejemplo, un aumento en la distancia de estas tierras no cambiaría el comportamiento de los campesinos desincentivándoles a estas prácticas, tampoco lo haría una 
suspensión de subvenciones. Es posible que la relación encontrada entre beneficios netos y los años de experiencia de los campesinos pudiera influir en la disminución en la práctica de agricultura itinerante con el paso del tiempo. Si las generaciones nuevas consideran estas prácticas como obsoletas podrían abandonar gradualmente la práctica de agricultura itinerante, teniendo como incentivo la obtención de mayores beneficios netos generados por la agricultura permanente. Este es un tema para futura investigación.

\section{Pagos por Servicios Ambientales}

Cabe preguntarse, sin embargo, si los incentivos para conservar y aumentar las superficies forestales son competitivos en comparación a los beneficios obtenidos por agricultura o ganadería. Los objetivos de los programas de pagos por servicios ambientales (PSA) son reducir la deforestación y reducir la pobreza mediante pagos a productores de servicios ecosistémicos. Si existen pequeños propietarios o comunidades en posesión de tierras con bosques o selvas podrían registrar un máximo de 300 hectáreas en contratos de cinco años en el acuerdo de no cambiar el uso de suelo y la cobertura forestal de la superficie establecida.

Teniendo en cuenta los pagos de las compensaciones registrados en PSA, el programa fijaba un pago de 1500 pesos por hectárea y por año en el 2012. Según los resultados de este estudio el beneficio neto aproximado por una hectárea bajo el sistema de agricultura itinerante y permanente sería, para el primer caso, un beneficio neto por hectárea es de unos 6700 pesos y en el segundo caso, de casi 12600 pesos a precios de 2012. Estos beneficios netos son reflejo de costes de producción e ingresos por ventas (o autoconsumo). En este caso la decisión de cambiar el uso de suelo a un uso forestal conllevaría la renuncia (o coste de oportunidad) de 5200 pesos por año y por hectáreas en caso de practicar agricultura migratoria y un coste de oportunidad de 11 100 pesos en caso de practicar agricultura permanente (maíz).

Otra alternativa que podría disminuir el valor del coste de oportunidad sería que, además, los beneficios netos pudieran estar compuestos también por costes y beneficios no monetarios propios (valores privados) o externos (externalidades), o ambos (valores sociales). En este caso, se analizaría si los valores no monetarios superan los valores estimados por este estudio reflejando un bienestar mayor a la renuncia de los beneficios netos monetarios obtenidos en la agricultura migratoria. 


\section{Conclusiones}

La pregunta sobre la posibilidad de aumentar los recursos forestales como una forma de mitigar el cambio climático podría no tener una respuesta definitiva debido a que hay algunas variables que intervienen. En muchos países los recursos forestales están en manos de comunidades locales que dependen de estos recursos y los usan para su sustento diario. Es posible que los propietarios (legales o no) de estas masas forestales no estén dispuestos a renunciar a los beneficios netos derivados de la explotación y consumo directo de recursos naturales.

En este estudio se analizan las características que podrían influir en la rentabilidad neta de sistemas de agricultura que pueden derivar en deforestación y degradación de bosques y selvas en el occidente de México mediante una regresión hedónica. Se encontró que los factores que influyen en los beneficios netos de la agricultura migratoria son la experiencia del campesino y la cantidad de tierra sembrada bajo este sistema de agricultura. Además, los estadísticos descriptivos muestran que la pobreza frecuentemente está presente en los procesos de degradación forestal (extracción de leña, talas ilegales, agricultura itinerante, etc.) y a menudo los beneficios obtenidos por estas actividades que degradan los recursos forestales no resuelven las condiciones de pobreza de las comunidades locales. La pobreza es también la principal causa de la práctica del sistema de agricultura itinerante, un sistema que requiere muy pocos insumos para su producción al ser una práctica rudimentaria basada en esfuerzo físico y un conocimiento del sistema de agricultura (años de experiencia).

Destacan también entre los resultados, que los beneficios netos obtenidos por campesinos que practican agricultura permanente, que son mayores (hasta cuatro veces) a los beneficios netos obtenidos por el grupo de campesinos que practican agricultura migratoria. Dado que estos beneficios netos incluyen el consumo de recursos naturales, se sugiere que los campesinos de mayores ingresos netos podrían consumir más recursos naturales con respecto a los campesinos de ingresos netos más bajos.

Por último, señalar que la noción de las comunidades locales sobre la conservación y protección de los recursos forestales de países en desarrollo pueden diferir a la noción de conservación de organismos internacionales y la comunidad científica. Un estudio que permita definir los lineamientos a seguir en conjunto con la población afectada, el conocimiento de las preferencias sociales y condiciones económicas podrían asegurar la participación de las comunidades locales. 
Economía Informa núm. 386 mayo - junio • 2014

En definitiva, el crecimiento de las superficies forestadas no es una panacea o una solución simple como forma de almacenamiento de carbono frente al cambio climático y el financiamiento de iniciativas internacionales tampoco deberían entenderse como la única fuente de desarrollo económico de comunidades rurales sino mas bien, como un complemento unido a otras iniciativas de los gobiernos locales y otras entidades.

\section{Bibliografía}

Adamowicz, W., Luckert, M., Veeman, M. (1997), Issues in using valuation techniques cross-culturally: three cases in Zimbabwe using contingent valuation, observed behaviour and derived demand techniques, Commonwealth Forestry Review, 76 (3): 194-197.

Miller, N. (2006), Notes on microeconomic theory, http://www.scribd.com/ doc/108489549/Lecture-Notes-on-Microeconomic-Theory-Nolan-MillersHavard

Brown, K., Barrows, R. (1985), The impact of soil conservation investments on land prices, American Journal of Agricultural Economics, 67: 1642-1650.

Dinar, A. Mendelsohn, R., Evenson, R., Parikh, J. Sanghi, A. Kumar, K., McKinsey, J., Lonergan, S. (1998), Measuring the impact of climate change on Indian agricultura, World Bank Technical Paper, núm. 402. Washington, D. C: The World Bank.

Ervin, D., Mill, J. (1985), Agricultural land markets and soil erosion: policy relevance and conceptual issues, American Journal of Agricultural Economics, 67: 938942.

Evenson, R, Alves, D. (1998), Technology, climate change, productivity and land use in Brazilian agricultura, Planejemento e Politicas Publicas, 18: 223-258.

FAO (2010), National deforestation estimates in percent and agricultural statistics. Global forest resources assessment, Main report. Food and Agriculture Orgnization of the United Nations, FAO. Forestry paper 163, Rome, Italy

IIED (2003), Valuing forests: A review of methods and applications in developing countries. Environmental Economics Programme, International Institute for Environment and Develpment, IIED.

INegr (2010), XlL Censo General de Población y Vivienda, Jalisco, México.

Mendelsohn, R., Nordhaus, W., and Shaw, D. (1994), The impact of global warming on agriculture: a ricardian analysis, American Economic Review 84:753-771.

Wunder, S. (2001), Poverty alleviation and tropical forests. What scope for synergies? World development, 29 (11): 1817-1833. 